\title{
Regards croisés et contacts entre syndicalistes français et allemands (1945-1962)
}

\author{
Sylvain SCHIRMANN
}

Il faut revenir dans quelques propos liminaires sur le premier après-guerre pour mieux situer la problématique d'un sujet consacré aux regards croisés et aux rencontres entre syndicalistes français et allemands au lendemain du second conflit mondial.

Après la Première Guerre mondiale, syndicalistes allemands et français se rencontrent exclusivement dans le cadre de leurs Internationales respectives (celle d'Amsterdam, ou l'Internationale chrétienne). Marqués par le conflit, les responsables y défendent souvent des positions très nationales (pour ne pas dire pas nationalistes) et se servent de ces structures soit pour remettre en question l'ordre issu des traités de paix, soit au contraire pour veiller jalousement à sa préservation. Jules Zirnheld, le leader de la CFTC (Confédération française des travailleurs chrétiens), ne se départit jamais d'une certaine méfiance à l'égard de l'Allemagne. Alors que les premiers congrès de l'Internationale chrétienne $(1920,1921)$ débattent de la pertinence des réparations, les représentants CFTC concèdent simplement que l'on puisse discuter éventuellement du montant. Ils se montrent intransigeants sur le principe des réparations, Jules Zirnheld décrivant avec passion au congrès de 1921 «le sol blessé du pays qui avait le malheureux et périlleux honneur d'être vainqueur». ${ }^{1}$ Dès 1922 Zirnheld obtient gain de cause en faisant adopter par l'Internationale chrétienne une motion interdisant toute immixtion dans le domaine des traités de paix. Cette intransigeance empêche pour longtemps toute rencontre directe entre les responsables de premier plan des organisations chrétiennes allemande et française. La première grande tentative de ce type a lieu le 19 mars 1932 à Aix-la-Chapelle. Elle débouche sur une promesse d'échange d'informations, mais vient, compte tenu du contexte, trop tard. ${ }^{2}$ Le refus de coopérer existe également du côté allemand. Le président de la Commission générale des Syndicats allemands n'a-t-il pas déclaré: «nous avons établi en substance une conception du travailleur qui n'existe dans aucun autre pays du monde», laissant entendre qu'il n'avait pas besoin de coopération internationale? ${ }^{3}$

Pourtant dans les deux pays des voix discordantes se font entendre. Gaston Tessier conteste par exemple l'intransigeance de Zirnheld, souhaite des rencontres régulières

1. Cité par G. LEFRANC, Les expériences syndicales internationales des origines à nos jours, Aubier, Paris, 1952, p.45.

2. Voir à ce propos J.-C. DELBREIL, Les catholiques français et les tentatives de rapprochement franco-allemand (1920-1933), Publications du Centre de Recherches Relations internationales de l'Université de Metz, Metz, vol.2, 1972. On se reportera aux pages 62 et suivantes pour les difficiles relations au sein de l'Internationale chrétienne, et aux pages 202 et 203 pour les contacts du début des années trente. On peut également lire J. ZIRNHELD, Cinquante années de syndicalisme chrétien, Spes, Paris, 1937.

3. Voir G. LEFRANC Georges, op.cit.; la citation est extraite de U. BORSDORF, Hans Böckler. Arbeit und Leben eines Gewerkschafters von 1875 bis 1945, Bund-Verlag, Köln, 1982, p.121. 
entre syndicalistes français et allemands, et espère à terme un rapprochement entre les économies et les systèmes de protection sociale des deux États voisins. ${ }^{4}$ Hans Böckler (le futur patron du DGB) dénonce quant à lui, dès les années vingt, «l'ampleur de la bêtise, de l'indifférence, de l'ignorance politique» de Carl Legien, dont les propos sont rapportés ci-dessus. ${ }^{5}$ Au cours de la seconde moitié des années vingt, les Internationales syndicales, celle d'Amsterdam comme la chrétienne, se prononcent à leur tour en faveur de la suppression des barrières douanières en Europe, et exigent des formes de coopération politique entre États. Point besoin d'insister sur l'échec de ces perspectives au cours de l'entre-deux-guerres. ${ }^{6}$

Le propos consiste alors à savoir si ce cours nouveau a pu s'imposer au lendemain du second conflit mondial. Si ces hommes qui accèdent en France comme en Allemagne aux plus hautes responsabilités syndicales (Tessier, Böckler), si ces partisans d'une coopération en Europe (Léon Jouhaux, par exemple) qui ont connu parfois l'expérience de la résistance et des camps ont donné une impulsion au dialogue et au rapprochement syndical franco-allemand. La méfiance qui existait au lendemain de la Première Guerre mondiale a-t-elle cédé la place à une certaine compréhension? Un premier fait est acquis: les rencontres entre syndicalistes français et allemands se sont multipliées après la Seconde Guerre mondiale. Un bilan de ces différents types de rencontre s'impose, pour mesurer pleinement les convergences entre les organisations nationales. Il serait incomplet, si on ne s'intéressait pas aux perceptions qu'ont les syndicalismes nationaux l'un de l'autre.

\section{Les rencontres entre syndicalistes français et allemands}

Les rencontres entre syndicalistes français et allemands furent fréquentes et régulières au cours de la période 1945-1962, même s'il faut signaler que les rencontres bilatérales directes furent moins nombreuses que celles qui s'effectuèrent dans un contexte multilatéral. Les contacts directs ont été nombreux de 1945 à 1950, au moment où les Alliés avaient la pleine responsabilité de l'Allemagne. Si Dwight D. Eisenhower encourage la reconstitution des syndicats dans une Allemagne débarrassée du nazisme, les Soviétiques sont cependant les premiers à les instituer dans leur zone, le 16 juin 1945. Les 17 fédérations qui se constituent à l'Est sont regroupées dans une organisation centrale, le FDGB (Freier Deutscher Gewerkschafts Bund). À l'Ouest, le mouvement est plus lent, et c'est à partir d'août 1945 que les circulaires envi-

4. J.-C. DELBREIL, op.cit., pp.103 sqq.

5. U. BORSDORF, op.cit., p.121.

6. Deux références pour cerner les débats des milieux syndicaux quant à un processus d'unification du continent européen pendant l'entre-deux guerres: W. LOTH, Von Heidelberg nach Godesberg. Europa-Konzepte der deutschen Sozialdemokratie zwischen Utopie und Politik, in: G. CLEMENS (Hrsg.), Nation und Europa. Studien zum internationalen Staatensystem im 19. und 20. Jahrhundert. Festschrift für Peter Krüger, Franz Steiner Verlag, Stuttgart, 2001, pp.203-219; D. GUERIN, Albert Thomas au BIT 1930-1932. De l'internationalisme à l'Europe, Euryopa, Genève, 1996. 
sagent la remise en route d'organisations syndicales. Les gouvernements militaires - c'est le cas des autorités françaises également - attendent des mouvements allemands qu'ils s'identifient à la politique des occupants, et qu'ils en soient un relais efficace. Les occupants espèrent un soutien au processus de dénazification, à l'éducation à la démocratie, et une approbation de la décartellisation. Autorisé à se reconstituer sur une base locale à partir de 1946, le syndicalisme allemand est confronté à un ensemble de problèmes: âge élevé des militants, dégâts des camps et confrontation à l'hitlérisme. Les militants ont en effet conscience d'une faillite antérieure qui a permis l'arrivée au pouvoir d'Adolf Hitler. ${ }^{7}$

Dans cette phase de reconstruction, les militants français, notamment ceux de la CGT (Confédération générale du Travail), sont sur place dès janvier 1946 pour recenser leurs homologues allemands, s'inquiétant du sort des survivants et aidant à la réinstallation des émigrés. Cette aide cherche aussi à diffuser les conceptions chères aux confédérations françaises. Léon Jouhaux explique par exemple en mars 1946 que la paix passe par «une économie dirigée et contrôlée de la nouvelle Allemagne». Pour le dirigeant français, cela suppose «remettre la gestion des entreprises mises sous séquestre entre les mains des travailleurs organisés». ${ }^{8}$ À la fin de l'année 1946, dans la zone française d'occupation (ZFO), le syndicalisme allemand a pris certaines caractéristiques françaises: une sous-syndicalisation par exemple par rapport aux zones britannique et américaine, et une plus grande division syndicale, trois organisations y coexistant, alors que dans les autres zones la tendance est au regroupement. Dès août 1946, un conseil syndical de la zone britannique s'est en effet constitué sous l'autorité d'Hans Böckler. Il se transforme en avril 1947 en DGB (Deutscher Gewerkschafts Bund), une confédération unique de la zone britannique. Un mois plus tard (mai 1947) se met en place un conseil syndical des zones britannique et américaine, que les organisations françaises rejoignent en novembre 1948. De cette structure sort, en novembre 1949, peu de temps après la naissance de la RFA, le DGB. À ce moment là, le taux de syndicalisation a progressé dans la ZFO, mais il reste toujours inférieur à ce que l'on observe en zone américaine ou britannique. ${ }^{9}$

Cette marche à l'unité ne heurte pas dans l'immédiat après-guerre les militants français. Au début de l'année 1946, Jouhaux, toujours lui, prenant acte de l'amorce du processus dans la zone britannique, en fait un modèle pour les autres zones:

«Aujourd'hui, écrit-il à la fin du mois de février 1946, [...] les militants ont conscience des tâches immenses que devra remplir le mouvement syndical pour rénover l'Allemagne. Aussi éduqués par les leçons du passé, conscients des effets néfastes que représenterait la pluralité syndicale, se sont-ils efforcés [...] de reconstituer les organisations sur la base de

7. Se reporter à H. GUNDELACH, Die Sozialausschüsse zwischen CDU und DGB. Selbstverständnis und Rolle 1949-1966, thèse, Bonn, 1983.

8. Cité par G. LEFRANC, op.cit., chapitre consacré à l'expérience syndicale allemande.

9. Les chiffres disponibles donnent les résultats suivants: $14 \%$ des salariés sont syndiqués dans la zone française; $24 \%$ en zone américaine et $25 \%$ en zone britannique. En 1949, le taux de syndicalisation est de $20 \%$ en ZFO, le double environ dans les zones britannique et américaine. On trouve des indications chiffrées dans G. LEFRANC, op.cit. 
l'unité, c'est-à-dire un seul syndicat groupant les ouvriers et les employés de la même industrie, sans distinction d'opinion politique et religieuse». ${ }^{10}$

Il faut dire également que le programme syndical discuté dans les assemblées qui précèdent l'unité du mouvement allemand offre, pour les syndicalistes français, des possibilités de convergence. Il y est question de démocratie économique et sociale, comme prolongement de la démocratie politique, de planification économique, de juste répartition de la richesse nationale, et d'amélioration des conditions d'existence, autant de points programmatiques pouvant convenir à des centrales françaises, qu'elles soient réformistes ou marxistes. La CGT a toujours une approche positive de l'unité syndicale allemande, même après la scission de 1947, car les syndicalistes communistes allemands, - à cause à la fois d'un militantisme actif et de l'héritage de Weimar - sont souvent surreprésentés dans l'appareil syndical Ouest-allemand. On le comprend bien à partir de l'exemple suivant: le parti communiste obtient 5,5\% des voix aux élections de Rhénanie du Nord-Westphalie en 1950, alors qu'au même moment $40 \%$ des délégués d'entreprise de ce Land sont membres du parti. La centrale française préfère d'autre part, comme la Fédération syndicale mondiale (FSM), la stratégie de présence dans des organisations puissantes au ghetto... Et en Allemagne cela permet, peut-être, un rapprochement à terme avec le FDGB de la zone soviétique. $^{11}$

Au fur et à mesure que l'unité syndicale avance en Allemagne, les échanges directs entre syndicalistes français et allemands s'espacent cependant. Les rencontres ont lieu à partir de 1948/1949 (naissance des deux États allemands) dans des cadres plus internationaux. Les travaux des Internationales fournissent des occasions d'échanges, l'OIT (Organisation internationale du travail) également. À partir de 1952, ce sont les structures de l'Europe communautaire (CECA, CEE), qui viennent s'ajouter à celles qui existent déjà. La CGT elle-même est confrontée à ce phénomène, ayant des contacts avec les responsables du FDGB dans le cadre de la FSM, ou des grands congrès à Berlin, Leipzig, voire Varsovie. La conséquence de l'évolution, c'est la constitution d'un groupe restreint de syndicalistes, de part et d'autre du Rhin, qui deviennent des «professionnels» de la coopération internationale et qui se rencontrent dans les structures qui organisent cette coopération. Émerge ainsi un noyau constitué d'hommes qui vont se croiser régulièrement dans ces instances au cours des années cinquante: Maurice Bouladoux, Roger Raynaud, Heinz Potthoff, Hans vom Hoff, Heinrich Deist... ${ }^{12}$

10. In G. LEFRANC, op.cit.

11. Ibid.; se reporter à la thèse de H. GUNDELACH, op.cit.; lire également B. FRACHON, Au rythme des jours. Rétrospective sur 20 années de lutte de la CGT (Textes choisis), tome premier, 1944-1954, Éditions sociales, Paris, 1967.

12. B. GEORGES, D. TINTANT (avec la collaboration de M.-A. RENAULD, Léon Jouhaux dans le mouvement syndical français, PUF, Paris, 1979. L'ouvrage permet de se rendre compte de l'activité internationale de Jouhaux. Ou encore T. REGIN, S. WOLIKOW (dir.), Les syndicalismes en Europe, vol.3, À l'épreuve de l'international, Syllepse, Paris, 2002, notamment l'article de T. REGIN, Les internationales syndicales en crise? (1945-1980), pp.81-98. 
À l'échelon de la base syndicale, les contacts entre responsables syndicaux français et allemands restent fréquents à deux échelons. Il s'agit d'abord de l'échelon des Fédérations internationales professionnelles, au sein desquelles on élabore, branche professionnelle par branche professionnelle, les revendications. On y coordonne l'action revendicative, en vue de l'obtention de «conventions collectives internationales, voire européennes». Il y est question de ce qui fait le quotidien d'un syndicaliste: salaires, conditions de travail, protection sociale... Dans ce cadre, par exemple, les responsables de l'IG Metall et des Fédérations françaises des ouvriers métallurgistes se retrouvent régulièrement. L'autre niveau de rencontres fréquentes, reste la zone frontalière. L'une des conséquences des rencontres entre militants lorrains et sarrois, c'est le maintien en Sarre d'un syndicalisme chrétien, qui bénéficie du soutien des syndicalistes chrétiens lorrains. Le type d'industrie (charbon, acier) de part et d'autre de la frontière, surtout avec la CECA, prédispose également à la multiplication des contacts dans l'espace sarro-lorrain. ${ }^{13}$

Les différents contacts, si l'on fait abstraction des discussions importantes sur les conditions de travail pour lesquelles les organisations souhaitent bien évidemment des améliorations, révèlent des convergences politiques qui suivent globalement le clivage CGT-FDGB d'un côté, syndicats réformistes (Force Ouvrière-FO, CFTC)DGB de l'autre.

\section{Convergences et divergences}

Ce clivage est évident sur les questions européennes. Du plan Marshall au traité de l'Élysée, FO, CFTC et DGB font quasiment la même analyse. Favorables au processus de la construction européenne tel qu'il s'est enclenché, elles n'en émettent pas moins des réserves quant au type d'Europe qui se met en place. Si elles souhaitent un renforcement des institutions européennes et davantage de supranationalité, c'est qu'elles estiment que c'est la condition d'une Europe plus sociale. Le traité de Rome marque ainsi, pour elles, un recul par rapport à la CECA. Cette Europe n'a de sens que si on l'élargit au Royaume-Uni et aux pays scandinaves (le fort taux de syndicalisation dans ces États et leur modèle de protection sociale ne manquerait pas espère-t-on - de favoriser l'émergence de l'Europe sociale). L'approfondissement de la construction passe également par une structure de planification européenne (cette revendication apparaît avec le traité de Rome). Un plan indicatif européen aboutirait à une politique européenne d'aménagement du territoire. Il s'agit là, pour ces orga-

13. On peut se rendre compte de l'importance des contacts entre fédérations professionnelles en se reportant à la biographie de Descamps commise par F. GEORGI, Eugène Descamps, chrétien et syndicaliste, Éditions de l'Atelier/Éditions ouvrières, Paris, 1997. Voir aussi: E. DESCAMPS, Militer. Une vie pour un engagement collectif, Fayard, Paris, 1971.

Pour le syndicalisme chrétien en Sarre: en 1956, au moment de sa fondation le syndicat chrétien allemand (CGB) compte à peu près 20.000 adhérents; en Sarre seule, le syndicalisme chrétien a 80.000 encartés (chiffres cités par Masses ouvrières, septembre 1956. 
nisations syndicales, de conditions pour une croissance qui irait de pair avec le progrès social. Cette Europe sociale reposerait sur une harmonisation de la protection et de la formation professionnelle. La construction d'une communauté de ce type dépend bien évidemment du moteur franco-allemand. Avec ce noyau réconcilié, elle contribuerait nécessairement à la paix. ${ }^{14}$

Quelques citations et actions éclairent bien ces positions. En 1949, Léon Jouhaux, dorénavant figure emblématique de FO, écrit par exemple:

«les syndicats français pourront étudier avec les syndicats allemands le régime qui doit être établi dans le bassin de la Ruhr pour qu'il cesse d'être un laboratoire de guerre et devienne une garantie de paix».

En mai 1950, le même Jouhaux insiste sur la nécessité d'

«une union économique franco-allemande qui rendrait toute guerre impossible entre les deux peuples et résoudrait la question sarroise ainsi que celles des limitations imposées à l'industrie allemande». ${ }^{15}$

De façon plus directe encore, Masses ouvrières, exprime, en octobre 1949 le même sentiment: «pour la France, il n'y aura pas de plus grande sécurité que l'Europe. Et de l'union France-Allemagne dépend toute l'Europe». ${ }^{16}$ En 1947, au congrès de Bielefeld, le DGB de la zone britannique insistait déjà sur une Europe unie, même si les contours de cette Europe restaient encore flous pour les responsables syndicaux. Les motions adoptées expliquent que «les peuples européens sont censés se réunir sous une forme ou une autre». On compte, à côté du moteur franco-allemand, sur «un processus quasi-inévitable qui devrait s'enclencher du seul fait des contraintes économiques». Les mêmes questions sont abordées au moment du congrès fondateur du DGB, à Munich en octobre 1949. Les textes élaborés alors insistent sur la nécessité de reconstruire des relations commerciales européennes, sur l'intégration de la jeune RFA «dans la communauté européenne des États démocratiques», cette dernière constituant à terme une troisième force entre les États-Unis et l'Union soviétique. Cette Europe émergera du seul fait «des changements structurels de l'économie mondiale» ${ }^{17}$.

14. S. SCHIRMANN, Les syndicats ouvriers français et les institutions européennes de la CECA aux traités de Rome: quelques approches, in: M.T. BITSCH (dir.), Le couple France-Allemagne et les institutions européennes, Bruylant, Bruxelles, 2001, pp.281-292; S. SCHIRMANN, L'Union départementale CGT de la Moselle face au plan Schuman, in: A. WILKENS (dir.), Le plan Schuman dans l'histoire. Intérêts nationaux et projet européen, Bruylant, Bruxelles, 2004 pp.185-196; W. BÜHRER, Les syndicats Ouest-allemands et le plan Schuman, in: A. WILKENS (dir.), op.cit., pp. 303-322; voir également T. REGIN, S. WOLIKOW, op.cit.; P. BROULAND, Les syndicats ouvriers français et l'Europe des Six, thèse de droit, Strasbourg, 1959; J. MEYNAUD, L'action syndicale et la Communauté économique européenne, Centre de recherches européennes, Lausanne, 1962; F. DEPPE (Hrsg.), Arbeiterbewegung und westeuropäische Integration, Pahl-Rugenstein, Köln, 1976; E.-D. KÖPPER Gewerkschaften und Aussenpolitik, Campus, Frankfurt/Main, 1982.

15. Cité par B. GEORGES, D. TINTANT, op.cit.

16. Masses ouvrières est la revue de l'Action catholique ouvrière. Cf. le numéro d'octobre 1949.

17. Cf. W. BÜHRER, op.cit. 
Le jeune militant Eugène Descamps partage le même point de vue. Revenant de Wlotho (près de Bielefeld) où il avait participé, avec René Rémond, à une rencontre de militants de l'Action catholique, il prend conscience, comme il l'explique par la suite, que l'internationalisme, la coopération et des relations étroites avec des mouvements étrangers, notamment allemands, ne constituent non seulement un impératif moral pour le mouvement ouvrier, mais que la prise en compte de la dimension internationale des phénomènes économiques et sociaux par le syndicalisme est une condition concrète de son efficacité. ${ }^{18}$ Ces prises de position soulignent les limites d'une action syndicale uniquement nationale: celle-ci ne correspond plus aux modifications intervenues au niveau économique. Il ne faut donc pas être surpris, qu'animés de telles réflexions, nombre de responsables syndicaux français et allemands adhèrent au Comité pour les États-Unis d'Europe de Jean Monnet.

CGT et FDGB, tout comme l'ensemble de la FSM, sont sur une ligne opposée. Faut-il rappeler la longue liste de griefs opposés par la CGT au plan Marshall, au traité CECA et au traité de Rome? Elle correspond à tout point de vue à celle du FDGB. Ces projets préparent la guerre d'agression du capitalisme, des magnats de la Ruhr contre l'URSS et les démocraties populaires. Ils marquent une étape de la paupérisation de la classe ouvrière. Dans ce contexte, la défense des salariés n'est efficace que dans un cadre national (faire pression sur les pouvoirs politiques nationaux). Il faut cependant maintenir une unité d'action entre les différentes confédérations nationales. ${ }^{19}$

La question du désarmement est plus complexe. CGT et FDGB s'opposent au projet CED (Communauté européenne de défense) et à toute forme de réarmement allemand. Les syndicats réformistes, en revanche, ont des positions moins uniformes sur ce problème que sur la construction d'une Europe unie. Si FO est favorable au projet de l'armée européenne, et de ce fait à la remilitarisation de l'Allemagne, il ne faut pas oublier que les minoritaires des Forces ouvrières syndicalistes européennes (FOSE) sont opposés au réarmement. À la CFTC, l'absence de prise de position officielle traduit la division du syndicat sur ces questions. Certains, résolument pacifistes, s'expriment dans Témoignage chrétien et se prononcent en faveur de l'unité d'action avec la CGT. D'autres, comme le groupe Reconstruction autour de Paul Vignaux, tout en étant contre une militarisation de l'Allemagne, ne veulent pas entendre parler d'une action commune avec la CGT et les communistes. Celle-ci renforcerait, à leurs yeux, l'URSS et le mouvement communiste, qui n'ont jamais réussi à fédérer librement le mouvement ouvrier. La question du réarmement allemand se

18. F. GEORGI, op.cit., pp.119-120.

19. Voir note 14, certaines références y figurent. Lire également, B. FRACHON, op.cit., tome 1er. 
réglera d'elle-même le jour où il y aura des élections libres dans une Allemagne réunifiée. ${ }^{20}$

Les embarras du DGB sur la question du réarmement se lisent à travers l'évolution de ses positions. Au début des années cinquante, la direction de la centrale est favorable au réarmement et à une contribution de l'Allemagne à l'effort de défense européen. Cela suppose pour l'Allemagne une égalité de traitement. Mais Hans vom Hoff ou Christian Fette sont contestés par leur base. Syndicats bavarois et rhénans amènent le DGB à faire marche arrière à son congrès de Francfort en octobre 1954. La centrale désapprouve la militarisation de l'Allemagne. Elle insiste dorénavant sur le fait que la paix se gagne avec des mesures socio-économiques. ${ }^{21}$

Autre élément, le projet syndical marque lui également des divergences et des convergences. Syndicat révolutionnaire et de lutte des classes, la centrale de Benoît Frachon souhaite, tout comme le FDGB de la RDA, la disparition du système capitaliste, dont le salarié n'a rien à espérer. En face le pôle réformiste, qui vise à la transformation du capitalisme, ne présente pas un visage homogène. Le réformisme du DGB est différent de celui des centrales françaises. Dès sa fondation, le DGB s'est lancé dans son combat en faveur de la cogestion. «C'est cela ou la lutte des classes» martelait Hans Jahn, du syndicat des cheminots. ${ }^{22}$ Le principe est simple: capital et travail doivent gérer à parité les entreprises. Lorsqu'en 1951 la loi sur la cogestion est adoptée en RFA, la CGT fustige ce «syndicalisme [qui] a abandonné la théorie socialiste révolutionnaire». ${ }^{23}$ Elle s'en prend à ces

«dirigeants du DGB [qui] se résignent plus que jamais au rôle d'un partenaire social défendant les intérêts limités de telle ou telle catégorie de travailleurs à l'intérieur d'un système général implicitement admis, et critiqué seulement dans des articles de presse ou des discours dominicaux». ${ }^{24}$

La cogestion est étrangère à $\mathrm{FO}$, pour laquelle le salarié n'a pas à participer à la gestion des entreprises. Le syndicalisme doit afficher son indépendance par rapport au patronat et à l'entreprise, comme par rapport aux forces politiques. Pour la CFTC, la cogestion dérive des principes d'organisation sociale du christianisme.

L'expérience intéresse le groupe Reconstruction pour lequel «la gestion économique de l'entreprise doit [également] revenir aux travailleurs organisés». Eugène Descamps lui-même emploie assez facilement le terme cogestion à partir de

20. Plusieurs brochures diffusées par la CGT méritent une attention particulière. Elles ont été parfois élaborées avec le Mouvement de la paix: La France devant les accords de Bonn et le traité de Paris, 1954, et La sécurité européenne et le problème allemand, 1955. Voir également: H. ROUSSEL, Force ouvrière et l'Europe, in: M. DREYFUS, G. GAUTRON, J.-L. ROBERT (dir.), La naissance de Force Ouvrière. Autour de Robert Bothereau, Presses universitaires de Rennes, Rennes, 2003, pp.233-245.

21. P. MEON, L'attitude des syndicats CGT, CFTC et DGB devant le problème de l'unification européenne 1950-1957, note de recherche, DEA d'Histoire sous la direction de Raymond Poidevin, IHEE, Strasbourg, 1982.

22. Cité par G. LEFRANC, op.cit.

23. Cahiers internationaux, juin 1956.

24. Cahiers internationaux, avril 1956. 
1952. ${ }^{25}$ À Reconstruction, et dans une plus large partie de la CFTC, on voit les avantages d'un tel système. Celui-ci est un outil de promotion de la classe ouvrière, car il nécessite une formation économique et la participation active de la base à l'action syndicale. On en vient alors à souhaiter une coopération plus approfondie sur cette question avec les autres syndicats en Europe. La cogestion suppose cependant une moindre étatisation de l'économie (le DGB l'admet en 1950), que la CFTC (y compris Reconstruction) a davantage de difficulté à rallier. La centrale chrétienne explique que l'État, par les nationalisations et par l'interventionnisme, est l'acteur clef pour traverser les aléas conjoncturels. Les pouvoirs publics créent ainsi les conditions de la prospérité économique, du progrès social et de la promotion des salariés. ${ }^{26}$ De telles approches permettent de mesurer les différences culturelles entre syndicats français et allemands, même si la volonté réformiste est présente dans les organisations de part et d'autre du Rhin. Cela rend parfois difficile l'unité d'action. Elle est également freinée par les regards que les deux mondes syndicaux portent l'un sur l'autre.

\section{Regards croisés}

Les organisations syndicales françaises envient la puissance du DGB autant qu'elles la craignent. Il est certain que le taux de syndicalisation, l'aisance financière et la capacité de mobilisation du DGB impressionnent les confédérations françaises. Assez régulièrement la presse syndicale française publie des reportages sur la centrale allemande, sur la nébuleuse de mutuelles et de coopératives qui tournent autour d'elle. Elle est fascinée par la durée de certaines grèves et par la capacité de mobilisation qui permet aux ouvriers allemands d'obtenir d'importantes hausses salariales. ${ }^{27} \mathrm{Ce}$ qui frappe encore les organisations françaises, c'est la capacité d'influence dans les questions européennes. Quel syndicat peut en effet se targuer d'avoir en la personne de Hans vom Hoff, un directeur au sein de la Haute Autorité de la CECA, en la personne de Rolf Wagenführ, le directeur du service statistique de la CECA, et surtout en la personne de Heinz Potthoff, un membre à part entière de la Haute Autorité. Potthoff est le second membre allemand de l'instance de la CECA, nommé à ce poste par Konrad Adenauer (ce qu'aucun syndicat français n'arrive à obtenir du gouver-

25. Cité par F. GEORGI, op.cit.

26. On peut se reporter à G. ADAM, La CFTC 1940-1958: histoire politique et idéologie, A. Colin, Paris, 1964; P. VIGNAUX, De la CFTC à la CFDT. Syndicalisme et socialisme: "Reconstruction» 1946-1972, Éditions ouvrières/Éditions de l'Atelier, Paris, 1980.

27. Par exemple, Recherches en décembre 1954, sur les banques du DGB, en juin-juillet 1957 sur le référendum syndical et les grandes grèves du Schleswig; cf. par exemple Syndicalisme CFTC, $\mathrm{n}^{\circ}$ 612, du 16 février 1957 sur cette capacité de mobilisation et les acquis qu'elle permet d'obtenir, à travers la même grève du Schleswig. 
nement pour un des siens). Les voies de l'influence sont ainsi démultipliées pour la centrale allemande. ${ }^{28}$

Les syndicats français ont alors des mots très durs pour ce syndicalisme de codécision. La CGT s'interroge tout d'abord sur la finalité de telles positions:

«On peut se demander si la bureaucratie syndicale n'a pas abusé l'opinion des masses [...] pour parvenir à s'assurer des fonctions lucratives dans l'économie. Ce syndicalisme donne naissance à une élite ouvrière qui partage avec l'élite des revenus élevés. Ce n'est ni plus, ni moins que le socialisme municipal appliqué à la société. La conséquence d'une telle attitude, c'est la pauvreté revendicative et doctrinale. Le DGB est marqué par un vide idéologique évident. Il traduit la disparition d'une élite spirituelle au profit d'une élite attachée à ses avantages matériels». ${ }^{29}$

Le DGB est ainsi accusé de «socialisme libéral». La cogestion constitue une erreur historique, répétition d'erreurs antérieures. Le DGB, lié au SPD, n'est-il pas l'héritier de mouvements qui ont voté les crédits de guerre en 1914, empêché la révolution en 1918/1919 et favorisé l'arrivée au pouvoir d'Hitler en 1933? La loi de 1951 se situe dans la continuité de ces abandons. ${ }^{30}$ Avec une ironie mordante, on parle alors de «ces managers de la cogestion» qui enchaînent le mouvement ouvrier au capitalisme. En 1960 encore, dans la presse cégétiste, on rend ce syndicalisme responsable de l'évolution du SPD au congrès de Bad-Godesberg. ${ }^{31}$

Le syndicalisme chrétien est lui également réservé sur certains aspects du DGB. La centrale allemande cumule pour la CFTC un double handicap: c'est une confédération socialiste dominée par les protestants qui se sont imposés aux catholiques. Dès lors la CFTC souhaite une remise en question de l'unité syndicale. Certains leaders CFTC expriment ouvertement leur incompréhension à l'égard de la stratégie de la CDU, acceptant ce monopole du DGB. Henri Meck, député MRP et responsable de la CFTC en Alsace, écrit à ce propos dans Syndicalisme en juillet 1950:

«les dirigeants socialistes ne respectent pas la neutralité. Pour les militants chrétiens, il aurait mieux valu évidemment reconstituer des syndicats chrétiens. Tôt ou tard, ils seront obligés de le faire». ${ }^{32}$

La CFTC n'a de cesse d'aider, soit directement, soit à travers la CISC à la reconstitution d'un syndicalisme chrétien en RFA. Elle soutient dès 1950 l'initiative de Arthur Grundmann (député FDP) qui remet sur pieds à Essen une Fédération des syndicats chrétiens. Seul un millier de personnes adhèrent au mouvement. Cela explique l'échec de la tentative. La constitution du CGB (Christlicher Gewerkschafts Bund - Confédération des syndicats chrétiens) en novembre 1955 a plus de succès. Vingt mille

28. Werner BÜHRER, dans son article Les syndicats ouest-allemands et le plan Schuman (op.cit.), présente ces différentes personnalités du syndicalisme allemand. Voir également D. SPIERENBURG Dirk, R. POIDEVIN, Histoire de la Haute Autorité de la Communauté européenne du charbon et de l'acier. Une expérience supranationale, Bruylant, Bruxelles, 1993.

29. Cahiers Internationaux, juin 1956.

30. Ibid.; voir également les propos d'Alain LE LEAP in: Cahiers internationaux, janvier 1956.

31. Cahiers internationaux, février 1960.

32. Cité par G. LEFRANC, op.cit. 
adhérents se recrutent en Bavière, en Rhénanie-Westphalie; le retour de la Sarre laisse espérer 80.000 membres supplémentaires. D'éminentes personnalités du monde catholique restent malgré tout opposées à cette création (Jakob Kaiser, Karl Arnold). ${ }^{33}$ Financée partiellement par la CISC, la nouvelle organisation a du mal à se démarquer du programme du DGB. Ne revendique-t-elle pas comme lui la cogestion? Les militants catholiques allemands préfèrent affirmer leur spécificité dans le cadre d'une organisation unique. La tentation identitaire ne les effleure pas. La CFTC considère parfois cette attitude comme un repli face au protestantisme. ${ }^{34}$

La scène syndicale française pose également quelques problèmes au DGB. ${ }^{35}$ Pour ce dernier, le modèle soviétique propagé par la CGT conduit naturellement à une impasse. La centrale allemande ne peut assimiler le socialisme à ce qui se passe dans les pays qui s'en réclament dans les années 1950. L'attitude de la CGT est synonyme d'absence d'unité syndicale, et le DGB considère la division syndicale française comme un archaïsme. L'erreur fondamentale, c'est le refus de voir l'évolution du capitalisme et les modifications qui se sont opérées au sein de la classe ouvrière. Celui-là dépasse de plus en plus le cadre national du fait des contraintes techniques; celle-ci a besoin d'un surcroît de compétences, de formation et de qualification. Dès lors les stratégies de l'affrontement n'ont plus de sens; elles doivent être complétées par des formes de participation à la gestion de l'entreprise, des territoires et de l'État. La coopération syndicale devient donc une nécessité, surtout dans le cadre européen. La division syndicale française est un frein, pour ce que le DGB espère: la participation à la gestion et à la politique du Marché commun.

Cela permet de comprendre pourquoi le DGB s'intéresse à ce qui se passe dans la minorité CFTC. De cette minorité et de Reconstruction pourrait surgir le renouveau $\mathrm{du}$ syndicalisme français. Ces différents groupes entendent créer les conditions du regroupement d'un syndicalisme de gauche, non communiste. Descamps, par exemple, aurait souhaité faire adhérer la Fédération CFTC de la Métallurgie à la Fédération internationale des ouvriers sur métaux (FIOM), proche de la CISL, à laquelle appartient IG Metall. Mais la menace d'exclusion que fait planer la CFTC l'amène à renoncer à son projet. ${ }^{36} \mathrm{C}$ 'est parmi les minoritaires de la CFTC que l'on réfléchit au socialisme démocratique, à la participation des travailleurs à la gestion des entreprises, à un plan européen, et que l'on développe des discours sur la formation ouvrière. Toutes ces réflexions intéressent le DGB. Elles constituent, pour la confédération allemande, une promesse, et ce d'autant plus que la CGT est obligée, au début des

33. H. GUNDELACH, op.cit.

34. Masses ouvrières, septembre 1956.

35. Cf. l'article de Karl BÖTTCHER sur les conceptions différentes du syndicalisme en France et en Allemagne, in: Masses ouvrières, janvier 1955.

36. F. GEORGI, op.cit. 
années soixante de prendre acte du Marché commun. La centrale proche du PC réclame en effet en 1962 l'union des forces dans le cadre communautaire. ${ }^{37}$

\section{Conclusion}

L'animosité qui prévaut souvent entre syndicalistes français et allemands au lendemain de la Première Guerre mondiale disparaît au lendemain du Second conflit. Les rencontres sont plus nombreuses, certaines positions convergent et les revendications catégorielles se rejoignent souvent. Malgré cela, les deux mondes syndicaux ont du mal à se comprendre. Modèles syndicaux français et allemand cherchent à s'adapter à l'évolution du capitalisme. Indéniablement le syndicalisme allemand parait avoir mieux réussi sa mutation. Son poids, son influence et la cogestion permettent à l'ouvrier allemand de bénéficier du plus haut niveau de vie, et souvent d'une meilleure protection sociale par rapport à son homologue français ou britannique. Divisé, replié sur des identités idéologiques et marqué par les stratégies de l'affrontement, le syndicalisme français a plus de difficultés. Des évolutions favorables s'y esquissent cependant. Le nombre de syndiqués progresse et des réflexions novatrices (cf. Reconstruction) y apparaissent.

La construction européenne représente un défi pour ces organisations. Elle oblige les syndicalistes (comme d'ailleurs les hommes politiques, les hauts fonctionnaires...) à des approches nouvelles. Cette période 1945-1962 prépare, à cause des contacts qu'elle a engendrés entre Français et Allemands, deux évolutions majeures du syndicalisme dans la décennie suivante: la naissance d'une confédération des syndicats européens et celle du premier Conseil syndical inter-régional, au niveau de la Lorraine, de la Sarre et du Luxembourg.

37. L'Humanité du 8 février 1962. Benoît Frachon explique qu'il «faut au sein du marché commun une union des forces et une réorientation nette de la lutte». Cela suppose, comme il le dit, une coordination des efforts de toutes les organisations. Cette prise de position a lieu au moment même où un mouvement d'ampleur se dessine en Allemagne de l'Ouest (Pays de Bade, Nord-Wurtemberg, ...) pour une augmentation salariale de $10 \%$. 